\title{
Arquitectura y "Cognoscimiento" Los Fenómenos Interpretativos involucrados en el Aprendizaje de los Procesos Proyectuales ${ }^{2}$
}

\author{
Adriana Masis Morales
}

\section{Resumen:}

En el campo de la Arquitectura, es común que las temáticas giren en torno a un proyecto (obra edificada o edificable), una intervención u otros objetos físicos y sus respectivos

Invitada nacional

Profesional Independiente

adrianama59@yahoo.es

Recibido: 22 de setiembre del 2016

Aceptado: 13 de noviembre del 2017

1 La palabra 'cognoscimiento' en este artículo hace referencia a un proceso en el que se accede al conocimiento a través de la cognición. Esta última viene a jugar un papel importante en el aprendizaje, pues es el factor diferenciador entre la información que no se aprende porque se percibe con indiferencia y la información que crece, se vincula, crea intereses, se cuestiona, confronta y trasciende su sentido inicial gracias a una percepción activa, que pone en valor las capacidades interpretativas de quien aprende. Esto permite una reacción y un involucramiento con la información, que finalmente se convierte en excusa para el desarrollo de múltiples razonamientos e inquietudes que invitan a continuar aprendiendo.

2 Este artículo surge a raíz del desarrollo y elaboración de la tesis titulada Hermenéutica de la Arquitectura: la relación sujeto-espacio interpretada desde los fenómenos poéticos y paradigmáticos, en donde a través del diálogo entre arte, arquitectura, literatura y filosofía, se vislumbra una serie de procesos interpretativos regularmente despreciados en las etapas previas a la materialización de una obra.

\section{Adriana Masís Morales}

Licenciada en Arquitectura en la Universidad de Costa Rica. Actualmente desarrolla proyectos e investigaciones de forma independiente. diseños, pues en la academia se tiende a inculcar que la labor del arquitecto está en dar soluciones a las necesidades de la sociedad a través de obras edilicias. Esto nos ha conducido a sobrevalorar lo tangible y los resultados, por encima de lo metafísico y los procesos, de manera que en ocasiones los estudiantes visualizan su quehacer arquitectónico como un acto mecánico, meramente práctico y enfocado en la obtención de un producto, más que en el aprovechamiento del proyecto de diseño, como herramienta de aprendizaje. A raíz de esta inquietud, surge la idea de reivindicar la importancia de lo cognitivo, representado en este ensayo a través de los conceptos de: el asombro, la experimentación y el compartir descubrimientos, como un medio para dar valor a las inquietudes intelectuales de los futuros arquitectos y de esta manera estimular el emprendedurismo y el auto-cuestionamiento; generándose un cambio en el paradigma de 'enseñar a través del método', a 'aprender a través de la experiencia'. Esto último quiere decir: dejar de visualizar el aprendizaje como una sucesión de lineamientos a satisfacer, para comenzar a fomentar el uso de valores cognitivos y vivenciales, ya que al concientizarse sobre ellos se da un acercamiento más integral a las variables implícitas en el diseño y se aviva el rol de los estudiantes, los docentes y el sistema educativo como constructores de nuevos conocimientos.

Palabras clave: aprendizaje; proceso creativo; asombro; experimentar; compartir conocimiento.

\section{Architecture and "Cognoscimiento"}

The Interpretive Phenomena Involved in the Learning of Project Processes

\section{Abstract:}

In the field of Architecture, it is very common that the topics revolve around a project (built or buildable construction), an intervention or other physical objects and their corresponding design, as the academic institutions tend to instill that the work of architects consists in giving response to the needs of society through built constructions. This has led us to overvalue the tangible objects and results, above the metaphysical aspects and processes, in such way that students, occasionally, visualize their architectural endeavors as a mechanical act, merely practical and focused on obtaining a product, rather than using the design project as a learning tool. In the wake of this concern, arose the idea to vindicate the importance of cognition, which is represented in this essay through the concepts of: amazement, experimentation and sharing discoveries, as a way to place value on the intellectual concerns of future architects, stimulating entrepreneurship and self-questioning, and generating a change in the paradigm of 'teaching through a method', to 'learning through experience'. The latter means to stop the visualization of learning as a sequence of guidelines to be satisfied and start the promotion of cognitive values and real-life experiences, since this awareness allows a more comprehensive approach to the implicit variables in design and fuels the student's, teacher's and educational system's role, as constructors of new knowledge.

Keywords: learning; creative process; amazement; to experience; share knowledge. 


\section{'Cognoscer' la Arquitectura}

iariamente, algunos profesores se enfrentan a dificultades en cuanto a la formulación de un sustento teórico-práctico que regule la manera de enseñar y evaluar; pero aun así, no muy seguido se reflexiona al respecto, ya sea por comodidad y conveniencia o por desinterés y falta de información, tanto por parte de los estudiantes, como por parte de quienes activamente participan en el diseño y ejecución de los planes de estudio. Inclusive, en ocasiones se parte de la noción de que 'cada quién aplica sus propios modelos de aprendizaje', pero, ¿cómo se dibuja un plan de la Arquitectura sin utilizar algún margen o regularidad? ¿Acaso existe una discrepancia significativa entre los criterios que definen el quehacer profesional y los criterios que moldean a los futuros arquitectos? Sea como fuera, es innegable que discutir constantemente al respecto y permitir a otras escuelas, profesionales y estudiantes dar retroalimentación sobre los logros y conflictos en el aprendizaje de la Arquitectura es un requerimiento para poder consolidarse como un área de estudio mucho más equilibrada y en armonía con los requerimientos de nuestro mundo cambiante.

Algunos profesores y especialistas en docencia universitaria aseguran que:

(...) se ha replicado una educación en compartimentos estancos llamados materias en donde se da más prioridad a los contenidos, como respuestas acabadas, que a los procesos.

[Y] En pro de la repetición de este modelo institucionalizado e internacional en el que se han venido creando lenguajes, se ha establecido una amnesia común hacia los propósitos pedagógicos. Las discusiones sobre la formación del arquitecto se han centrado básicamente en esta misma "compartimentación", y las preocupaciones se debaten entre la intensidad horaria de una materia, o el semestre en el cual debe ser vista. (Castaño, Bernal, Cardona \& Ramírez, 2005, p.132)

Es claro que el aprendizaje de laArquitectura requiere todo un espectro de conocimientos que, actualmente, a nivel académico, se encuentran enmarcados bajo el nombre de cursos; sin embargo, sería de gran provecho dejar de lado el carácter radio-centrista de cada curso, pues finalmente esto contribuye a una percepción del conocimiento "por islas" que, como dice el arquitecto Óscar Guevara refiriéndose a las temáticas de estudio, "conviven juntas, pero sin interactuar" (2013, p. 33). Cada curso busca tomar un papel protagónico que menosprecia o ignora la existencia de todos los demás como posibles complementos. En ese caso, habría que advertir, al menos, al estudiante sobre los muchos usos de esos conocimientos y que es un deber de todos los allegados al mundo de la Arquitectura descubrir las interacciones entre esos conocimientos.

Es necesario recalcar en este punto que al hablar de 'advertir a los estudiantes' debe entenderse: instarlos a concientizarse sobre las realidades de sus localidades y del mundo, sobre la existencia de criterios compatibles y contradictorios, así como promover que opinen, se expresen y actúen con fundamento. El arquitecto Helio Piñón, quien comenzó su carrera docente a comienzos de los años setenta en la Escuela Técnica Superior de Arquitectura de Barcelona y hasta la actualidad se mantiene investigando, opina sobre las mejores prácticas para la transmisión del conocimiento y advierte que, al no incentivar la formación de criterios, las escuelas de Arquitectura corren el riesgo de convertirse:

(...) en guarderías de jóvenes de edades comprendidas entre los 18 y los 25 años, fascinados por un presente "creativo", a la espera de un futuro como "estrellas" con una popularidad comparable a la de un deportista o un cantante. [Y agrega, apesadumbrado, que] la realidad [desde su punto de vista,] es que nuestras escuelas están formando mano de obra barata para los grandes estudios multinacionales que han convertido el proyecto en una actividad industrial que actúa con criterios estéticos propios de un populismo banal y con procedimientos publicitarios propios del mercantilismo más burdo. (Piñón, 2007, párr. 16)

Es difícil concordar de manera absoluta con estas sentencias pues, primero, hay estudiantes con gran capacidad intelectual y de discernimiento, y arquitectos/docentes muy comprometidos con su profesión, que demuestran ser excepcionales, desmintiendo el alegato de Piñón, y en segundo lugar, porque, aunque las escuelas representan gran parte del proceso de formación, a fin de cuentas no son las únicas responsables. 


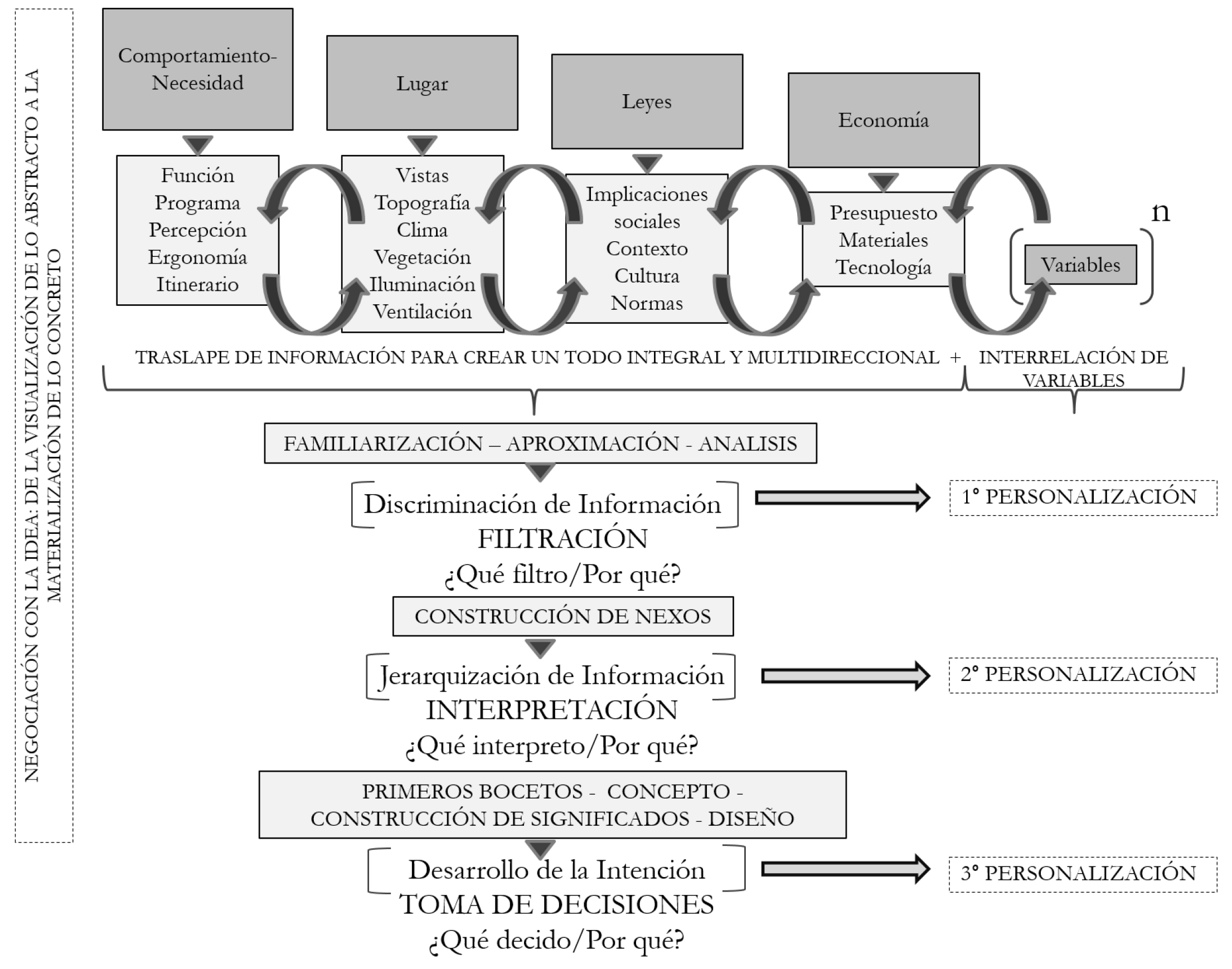

A

Figura 1. Diagrama Personalización del Proceso Creativo. Elaboración propia.

3 El taller se define como la modalidad pedagógica comúnmente utilizada para el aprendizaje del diseño arquitectónico, que simula el ejercicio práctico profesional de resolución de proyectos y constituye la columna vertebral para la experimentación, cuestionamiento y aplicación integral de los conocimientos adquiridos en los demás cursos que componen la malla curricular de la carrera de Arquitectura.

$4 \quad$ El arquitecto Óscar Guevara en su tesis doctoral titulada: Análisis del Proceso de Enseñanza Aprendizaje de la Disciplina Proyecto Arquitectónico, en la Carrera de Arquitectura, en el Contexto del Aula (2013) muestra el concepto de 'enseñanza' de la Arquitectura como un complemento que es, a la vez, opuesto al concepto de 'aprendizaje' de la Arquitectura, en donde el hecho de que existan modelos de enseñanza consolidados no significa, según su investigación, que se pueda enseñar la Arquitectura, sino más bien crear procesos de aprendizaje. De este modo, cuando se habla de la 'enseñanza' dentro de este artículo, se refiere a la modalidad que se rige estrictamente por métodos científicos tradicionalistas, y cuando se habla de 'aprendizaje', se refiere al uso de procesos alternativos que rompen la circularidad epistémica y dinamizan la creación de conexiones mentales en quienes aprenden.
También, se ha llegado a pensar que si tal compromiso y madurez mental de integrar los conocimientos depende de cada estudiante y la forma en que experimenta su paso por la carrera de Arquitectura, entonces se trata de una vivencia tan personal como privativa, por lo que sería imposible para un estudiante transferir su particular y única resolución de ideas a otro. Comúnmente observamos que ante un problema de diseño existen tantas respuestas como estudiantes en el taller ${ }^{3}$. Ninguna es igual o tiene los mismos valores que la otra, y eso quiere decir que gran parte del proceso se resuelve a través de discusiones mentales del alumno consigo mismo. Es por esto que también existe una postura contraria a la de la enseñanza de la Arquitectura ${ }^{4}$, que dice que:

[Ser arquitecto] no depende de la discusión sobre ninguna definición, ni sobre alguna supuesta formación para llegar a ello, sino que simplemente es una vivencia personal e intransferible, una aspiración vital, una realización de la propia existencialidad, que no depende tanto de una formación como de una visión: "Arquitectura es visión y por tanto es imposible de enseñar y difícil de aprender." (Simposio 2005 citado en, Guevara, 2013, p. 32)

Evidentemente, toda persona que se dedica a la Arquitectura experimenta en su proceso un tiempo de negociación de la idea, en el cual, más allá de la objetividad con la que quiera resolver su proyecto, realiza actos que personalizan el proceso (ver figura 1). Recordemos que, según apuntan los arquitectos Eva Álvarez y Carlos Gómez en su Crítica Radical a la Enseñanza de la Arquitectura; "no existe el análisis objetivo ya que la elección de las variables a estudiar presupone una toma de partido previa, que habría que argumentar" (Álvarez \& Gómez, 2009, p. 9), y esto hace que exista una pluralidad de resultados ante una misma situación.

De hecho, el profesor y arquitecto de la Universidad Politécnica de Cataluña, Alfred Linares (2006, p. 57), menciona que la singularidad es una cuestión esencial para la Arquitectura. Y así, lo que se filtra, interpreta y decide, parecen ser cuestiones 
indefinibles que impiden la existencia de un método único para trabajar la Arquitectura, pero que son procesos mentales que se debe promover que los estudiantes aprendan a reconocer por su cuenta.

Tal y como se observa en la figura 1, las distintas etapas de la dinámica del proceso creativo no constituyen elementos aislados, pues todas ellas se traslapan y se comportan como un todo. Los procesos realmente no son unidireccionales, como los expone el modelo mecanicista, sino que comprenden la posibilidad de retornar en sí mismos y llegar a resultados variados. Las actividades propias del proceso no terminan para que comience la siguiente, sino que se transforman en la siguiente, de manera progresiva y plural, según las experiencias y modelos de conocimiento que se vayan manifestando.

A propósito de esto, el arquitecto y profesor José A. Martínez Lapeña comenta: "(...) me parece que los alumnos van a recoger de distintos profesores, que están pasando por la escuela, una serie de datos, de ideas, de actitudes diferentes frente a los mismos problemas" (Martínez, citado en Conte-pomi, 2009, p. 331).

Por lo tanto, si el aprendizaje de la Arquitectura es algo que se da en medio de pluralidad de cursos, pluralidad de matices dados por los profesores en cada época, pluralidad de vivencias a lo largo de la carrera, y pluralidad de estudiantes que aspiran a descifrar el valor (o los valores) pregnante(s) de las llamadas obras arquitectónicas, entonces eso implica que intentar regular ese aprendizaje es algo altamente difícil, pero no imposible. La imposibilidad está en el enfoque que se ha manejado hasta ahora, pues el lenguaje nos limita a entender la enseñanza como algo que se imparte al alumno como colocar información en un archivero. Bajo la idea de la enseñanza se entiende que se imparte una lección y esa lección se incorpora al campo de inmanencia del estudiante. Es decir, las ideas se agotan en sí mismas en la mente del que es enseñado.

La palabra enseñanza tiene una gran carga sobre esto. Si se busca en el Diccionario de la Real Academia, se encuentra que enseñar significa: "instruir, doctrinar, amaestrar con reglas o preceptos, dar advertencia o escarmiento, habituarse 0 acostumbrarse a algo" (Real Academia Española, 2014). Se trata de una palabra que, veladamente, da a entender que el docente alecciona sobre algo que ya está dado, reglamentado y que solamente se puede hacer de cierta manera, a la que el estudiante debe habituarse. Entonces, bajo el entendido de que esta profesión se mueve en el ámbito de la multiplicidad, valdría la pena hablar mejor de los procesos para el "cognoscimiento" de la Arquitectura, en vez de adoptar únicamente el concepto de enseñanza, ya que, en este último, muchas veces la dinámica se restringe a un ritual en el que el alumno se equivoca y el profesor corrige.

Referirse a un "cognoscimiento" implica conocer la Arquitectura desde la cognición. Es decir, que la información dejaría de ser un rasgo inmanente al estudiante y se convertiría en algo trascendente. Esto significa partir de que las ideas, conceptos y temas en general no se archivan en la mente y se mantienen inmóviles, sino que hay un procesamiento, una interpretación y una experiencia de por medio que engrandecen el conocimiento.

\section{Asombrarse, experimentar y compartir descubrimientos: las bases para construir un proceso de aprendizaje}

En la actualidad, al aprender Arquitectura vivimos en una pluralidad de enfoques: puede suceder que en un curso todo pertenezca a un orden funcionalista, pero en otro hay un rigor historicista, y en otro el valor recae sobre lo perceptual. Algunos se preocuparán por mostrar la Arquitectura de las geometrías puras y los números, otros por lo orgánico, otros por lo técnico-constructivo, otros por lo simbólico o lo ambiental y otros por lo tecnológico y las nuevas ponencias de los arquitectos de vanguardia. Evidentemente, no sería esperable que en el curso de construcción, se enseñe paisajismo, y cada curso representa para el estudiante una pieza más del gran rompecabezas mental que intenta resolver a lo largo de su aprendizaje.

Entonces, ¿cómo se construye este rompecabezas?, ¿de cuántas dimensiones es o cómo se unen las piezas?, ¿cuáles piezas van primero?, ¿tienen todas la misma 
jerarquía?, ¿llevan a todos al mismo resultado? Primeramente, el rompecabezas del aprendizaje se comienza a construir una vez que el estudiante se da cuenta de lo que tiene que construir; sus dimensiones dependen de las habilidades asociativas de los estudiantes (proceso en el que contribuyen fuertemente los profesores) y la cantidad de piezas; orden y jerarquía no son primordiales, pues pueden variar indefinidamente, siempre y cuando el estudiante sustente y reflexione su proceso de aprendizaje sobre las bases del asombro (primer motor del conocimiento), el experimento progresista (proceso transformador) y el descubrimiento compartido (resultado expuesto).

Pero, ¿por qué se puede considerar a estas tres como las bases para un proceso de aprendizaje y desarrollo de ideas? Ciertamente, el asombro se puede visualizar como un motor del conocimiento que nos llevamos cuando hacia nuestros adentros decimos: "no tenía idea de esto", "esto es nuevo para mí" o "nunca lo había pensado de esta manera". Quiere decir, que representa aquel momento en que caemos en cuenta de algo desconocido, que genera curiosidad y extrañamiento a la vez. Es además una muestra de que lo que sucede a nuestro alrededor no nos es indiferente, pues nos hemos concientizado sobre una situación particular y se ha despertado un interés que invita a acceder a información que se desconocía. De hecho, tal y como lo exponen Platón y Aristóteles, la admiración (o asombro) es el origen del filosofar, y sabiendo que filosofar implica cuestionar, argumentar, asociar ideas y crear conocimientos (entre muchas otras cosas), se puede decir que quien presta atención al mundo y es sensible a los sucesos del mundo, abre su mente a la posibilidad de aprender y generar nuevas ideas.

Ahora bien, en compañía del asombro se halla el experimento progresista, el cual viene a representar un accionar que permite sacar conclusiones de lo aprendido. Si únicamente se diera el asombro sin hacer nada al respecto, el aprendizaje se estanca, pues el acto de percatarse de algo o admirar algo es por sí solo un acto pasivo; sin embargo, al tratar de crear algo con aquella información recién adquirida, se da la oportunidad de analizar, contextualizar la utilidad de estos nuevos conocimientos y comenzar a tener criterios válidos, que se basan en lo que hemos experimentado. Por otro lado, en este concepto no sólo se habla del experimento, sino que se dice que es progresista, pues el acto de crear por sí mismo, no necesariamente implica que hubo un crecimiento en el aprendizaje. La capacidad para generar ideas, llevar a cabo nuevas propuestas y extender aún más nuestro abanico de conocimientos, es concebible sólo cuando el experimento no es un fin en sí mismo, sino que constituye un proceso para transformar pensamientos y continuar obteniendo una comprensión de la Arquitectura, cada vez superior y más compleja, que progresa y va mutando a lo largo del tiempo.

En efecto, tanto el asombro como la experimentación progresista son actos que, a pesar de que tienden a pasar desapercibidos cuando se habla del proceso creativo y del aprendizaje de la Arquitectura, conforman una parte muy importante de la formación de los arquitectos, ya que tienen mucho que ver con la manera subjetiva en que cada individuo razona e interpreta sus vivencias particulares, y con los posibles intereses, proyectos y propuestas que realizará en su vida profesional.

A partir de esta afirmación se comprende que el asombro y el experimento progresista forman parte del proceso de aprendizaje de una manera un poco más individual. Pues, entre quienes nos dedicamos a estudiar y aprender de la Arquitectura, la mayoría probablemente no nos asombramos por las mismas cosas, ni decidimos tomar las mismas acciones para experimentar con lo que aprendemos. Como se mencionó antes, existen múltiples resultados y propuestas para una misma problemática, y la tercer base del aprendizaje mencionada trata de sacar provecho de esta diversidad de ideas. El descubrimiento compartido hace hincapié sobre la necesidad de evitar que los estudiantes no vean más allá de sus propias ideas y tengan la oportunidad de conocer distintos enfoques creativos y modos de entender la Arquitectura. Básicamente, se trata de tomar el proceso creativo individual, para exponerlo, debatirlo, y que el conocimiento que pertenece a unos pocos, se multiplique y re-interprete a través de la confrontación con las ideas de otros. Esto no sólo permite engrandecer el aprendizaje a través de la experiencia, sino también promover la activación del proceso infinito de asombrarse, experimentar y compartir descubrimientos. 


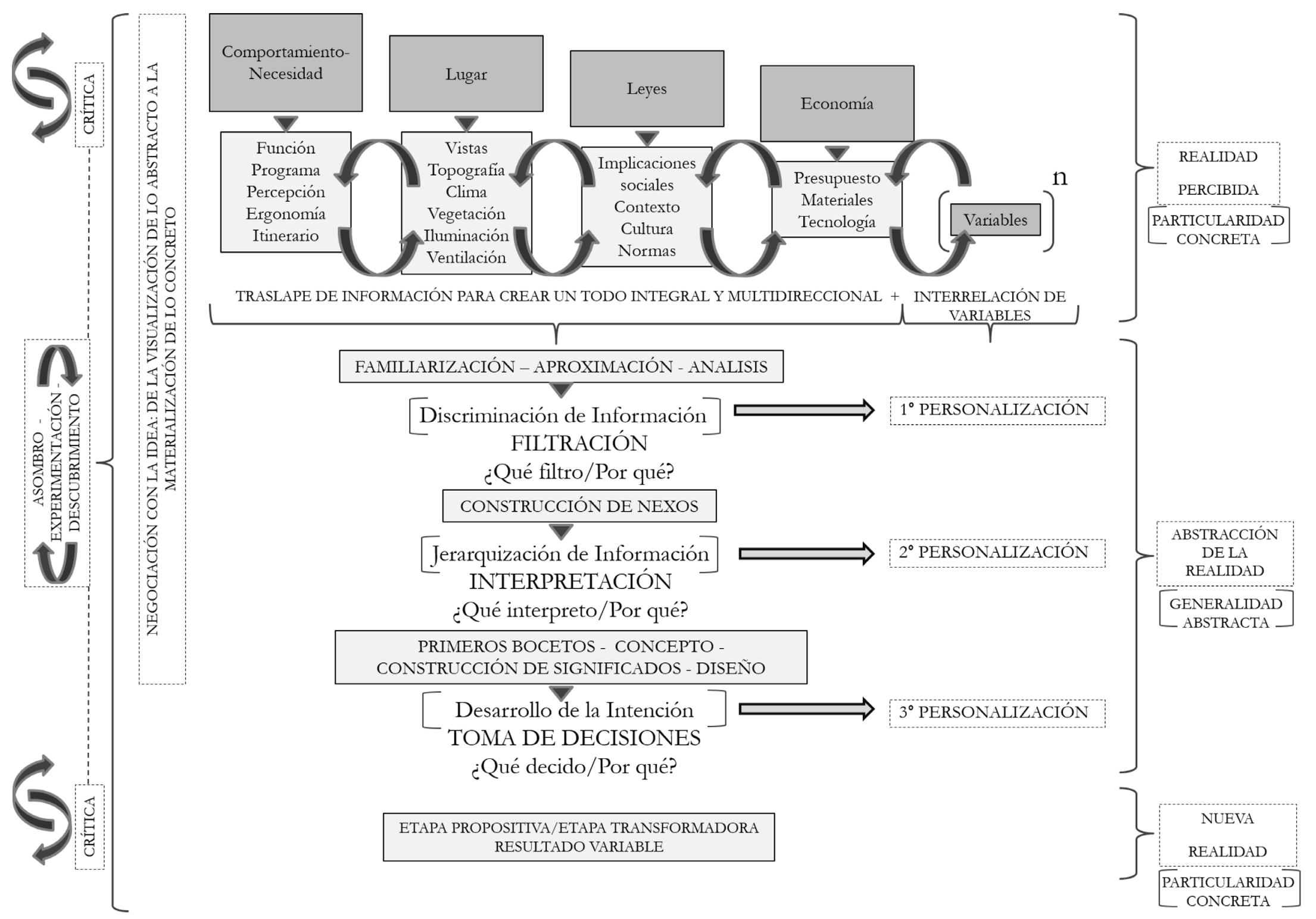

A

Figura 2. Diagrama Yo-Proyecto (enfocado en la idea de Proyecto). Elaboración propia.
Este diagrama muestra cómo el proceso en el que se trabaja una idea está contenido dentro de una circularidad epistémica que se compone del asombro, la experimentación y el descubrimiento, como motores fundamentales para el desarrollo de las ideas y que, mediados por la crítica -constante de principio a fin- permiten llegar a los distintos resultados, que rompen esa circularidad. A continuación se profundizan y amplían cada una de estas bases para el proceso de aprendizaje y desarrollo de ideas.

\section{Primer Motor: El Asombro}

El asunto del conocimiento, necesariamente, es un asunto asociado al desconocimiento y el asombro surge cuando hacemos conciencia de aquello que es desconocido (o conocido y percibido de una manera nueva). Ahora bien, para saber que sabemos, antes hemos atravesado una etapa de no-saber, y probablemente cada nueva cosa que sabemos, abre la puerta a nuevos no-saberes por explorar. El distinguido arquitecto Steven Holl en una entrevista comentaba: "Los estudiantes tienen que trabajar con duda e incertidumbre. Tan pronto como sepas qué es la Arquitectura estás taponando muchas posibilidades" (Moya-Angeler, 2007, p. 85-86).

Ciertamente, todo lo desconocido inspira incertidumbres y dudas, pero una vez que se aprende a sacar provecho de tal estado, las nuevas preguntas y respuestas nos llevan al asombro. Nuestra capacidad de asombro ha fundado un quehacer imaginativo en la Arquitectura que nos permite observar el mundo con una mirada más atenta y extraer el sentido de la cúpula, al mirar las montañas, o entender la función de un tirante, al ver las lianas, y el principio de arco o el de bóveda, al ver las cuevas.

El conocimiento parte de una motivación epistemológica que nos estimula y nos insta a hacernos preguntas, buscar problemas y curiosear los motivos de las cosas; y si fuéramos indiferentes a todas estas incitaciones, no existiría un punto de partida para crear y los días pasarían estoicamente, sin dejar rastro de su existencia. Esta noción sobre la ausencia de asombro, es lo que expresa la poetisa Wisława Szymborska en su poema titulado 'Falta de Atención' que dice: 
Ayer me porté mal en el cosmos.

Viví todo el día sin preguntar por nada,

sin sorprenderme de nada.

Realicé acciones cotidianas,

como si fuera lo único que tenía que hacer.

Aspirar, respirar, un paso tras otro, obligaciones,

pero sin pensamientos que fueran más allá

de salir de casa y volver a casa.

El mundo podría ser tenido por un mundo loco

y yo lo tuve para mi propio y trivial uso.

Ningún cómo, ningún por qué,

0 de dónde ha salido éste,

o para qué quiere tantos impacientes detalles.

Fui como un clavo superficialmente clavado a la pared,

(aquí una comparación que no se me ha ocurrido).

Uno tras otro se fueron sucediendo cambios

incluso en el limitado campo de un abrir y cerrar de ojos.

En la mesa más joven, con una mano un día más joven

había pan de ayer cortado de forma distinta.

Las nubes como nunca y la lluvia como nunca,

porque era con otras gotas que llovía.

La Tierra giraba sobre su eje

pero en un espacio abandonado para siempre.

Duró sus buenas 24 horas.

1.440 minutos de ocasiones.

86.400 segundos que mirar.

El cósmico savoir-vivre

aunque calla sobre nuestro asunto,

exige, sin embargo, algo de nosotros:

una cierta atención, un par de frases de Pascal

y una sorprendente participación en este juego

de reglas desconocidas.

(Szymborska, 2009, pp. 32-33)

La atención que requieren de nosotros los fenómenos del mundo es la atención propia de la pureza intelectual que les permite a los niños cuestionarse aspectos de la vida que los demás dan por hecho y hacer sus propios descubrimientos sobre los significados de las cosas. El conocimiento siempre está presente; no está exclusivamente plasmado en los libros y las enciclopedias, o en las personas con mayor cantidad de diplomas. El conocimiento excede todos estos objetos y personas, y se manifiesta a quienes son capaces de librarse de la prepotente sabiondez, buscan incansablemente las verdades de las cosas y aprenden a despreocuparse de ser considerados ingenuos en sus búsquedas y experimentaciones, ya que persiguen un apetito por la construcción de ideas y pensamientos que requiere constancia, profundización y, en definitiva, capacidad de asombro.

Aristóteles, al inicio de su Metafísica, comenta: "Todos los hombres [seres humanos] por naturaleza desean saber. Señal de ello es el amor a las sensaciones" (Aristóteles, 
1994, p. 69). Y, ciertamente, los sentidos son nuestra manera más elemental de saber que estamos en el mundo y lo que nos permite absorber información que luego será traducida en preguntas que buscan la información menos evidente que escapa a nuestros sentidos.

La constante búsqueda del asombro o admiración es lo que marca esa diferencia entre mantenerse satisfecho con los conocimientos ya dados (los que están presentes en la superficie) o tomar el protagonismo de construir nuevos conocimientos. Por ejemplo, en el diálogo Teeteto de Platón se habla de que Iris es hija de Taumas. Iris representa la ciencia y la filosofía como hijas de Taumas, que significa asombro (Platón, 1871, p. 182). En ese sentido, el asombro viene a ser como un descubrimiento de algo ordinario, transformado ante nuestra presencia en algo extra-ordinario; lo cotidiano llevado a otro nivel que nos coloca en otro estado de ignorancia. Es decir, el asombro se convierte en el conocimiento de algo nuevo que a la vez abre la puerta a una gama de no-saberes.

No obstante, el no-saber es algo usualmente mal visto por la sociedad, a pesar de que todos somos ignorantes en alguna medida. Pero, aunque culturalmente se pueda considerar vergonzoso, es en el reconocimiento de los no-saberes que se instalan las ansias por des-cubrir y de-velar, tras lo común y lo preestablecido, nuestra pequeña gloria innovadora.

El conocimiento de esta forma, actúa como un ciclo interminable de preguntas entre las cuales se llega a algunas respuestas; pero, más que nada, nos introducen a más preguntas. Heidegger, refiriéndose a la naturaleza indagadora de la Filosofía, apunta: "Nuestro preguntar sobrepasa lo habitual y lo ordinario y bien ordenado en el espacio cotidiano. (...) [Quien busca el conocimiento] vive, ve, oye, sospecha, espera y sueña constantemente cosas extraordinarias" (Heidegger, 2001, p. 21).

Sin embargo, así como se teme a reconocer el asombro para no reconocer la ignorancia, en los procesos proyectuales el papel en blanco literalmente representa una pesadilla del no-saber. La hoja en blanco sirve como analogía del asombro, pues expresa aquel momento en el proceso de diseño en que nos percatamos de que tenemos muchas preguntas ante algo inexplorado y de lo que no sabemos con exactitud cuál será el resultado final. Según esta percepción, es el momento que antecede a las primeras ideas, pues se supone que esa hoja en blanco es una metáfora del estado de la mente del arquitecto antes de comenzar su diseño.

Tal cosa tiene poco de verdad, ya que rara vez (por no decir nunca) empezamos de cero. La mente de un arquitecto siempre está llena de ideas, circunstancias, problemáticas; y una hoja, aún vacía, representa increíbles posibilidades para el asombro. Es la oportunidad justa para asombrarse de lo que se conoce, lo que se desconoce y lo que se cree que podría ser.

Edgar Allan Poe se cuestiona sobre la gran omisión de explicaciones por parte de los artistas y autores que evaden explicar sus procesos creativos en el momento en que se enfrentan al papel, por lo que en su Filosofía de la Composición relata:

He pensado a menudo, cuán interesante sería un artículo escrito por un autor que quisiera -considerando que pudiera- describir, paso a paso, los procesos seguidos en cualquiera de sus obras hasta llegar al término definitivo de su realización. A pesar mío, me sería imposible explicar por qué no se ha ofrecido nunca al mundo un trabajo semejante; pero quizá la vanidad de los autores haya sido la causa más poderosa que justifique esa laguna literaria. Muchos escritores (especialmente los poetas) prefieren dejar creer a la gente que escriben gracias a una especie de sutil frenesí o de intuición extática; pero experimentarían verdaderos escalofríos si tuvieran que permitir al público echar una ojeada tras el telón, para contemplar los trabajosos y vacilantes embriones de pensamientos (...) [traducción propia]. (Poe, 1846, p.1)

Usualmente, vemos los croquis de la primer idea de un arquitecto o vemos inclusive a los artistas y poetas, que pareciera que mágicamente consiguen envolverse en un torbellino de ideas que los lleva a diseñar, pintar y escribir fluidamente como si fuera la cosa más natural del mundo, mientras nosotros, en ocasiones, nos sentimos desprovistos de esta habilidad. Pero, ¿alguna vez reflexionamos sobre el proyecto antes de enfrentarnos al papel? Esto quiere decir que probablemente en nuestro apuro por declarar nuestras intenciones y demostrar nuestras capacidades, acudimos al papel antes de tiempo. Por 
esto, el asombro ante lo desconocido (como la hoja en blanco) no debería ser temido, sino más bien una motivación ante todo el nuevo conocimiento que se está a punto de adquirir.

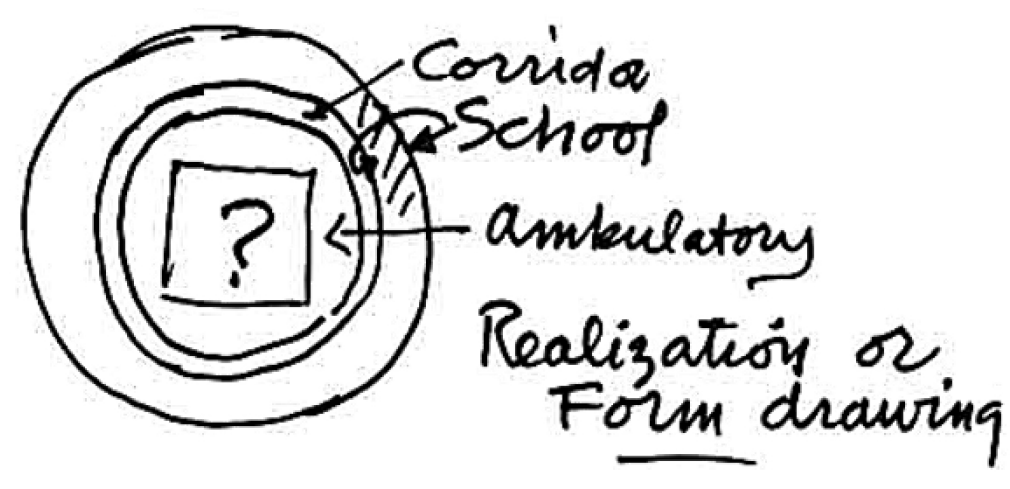

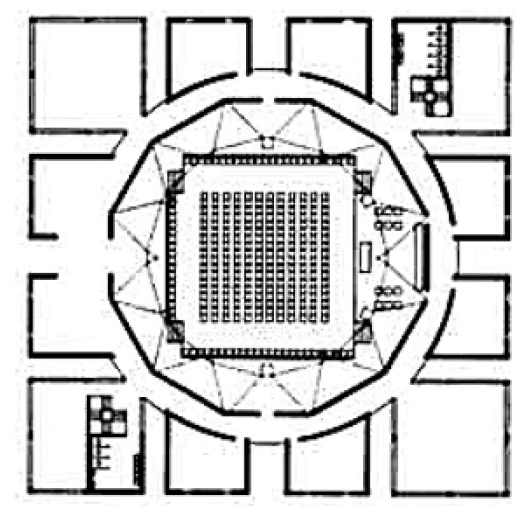

FIRST DESIGN close tranelation of realization in Form

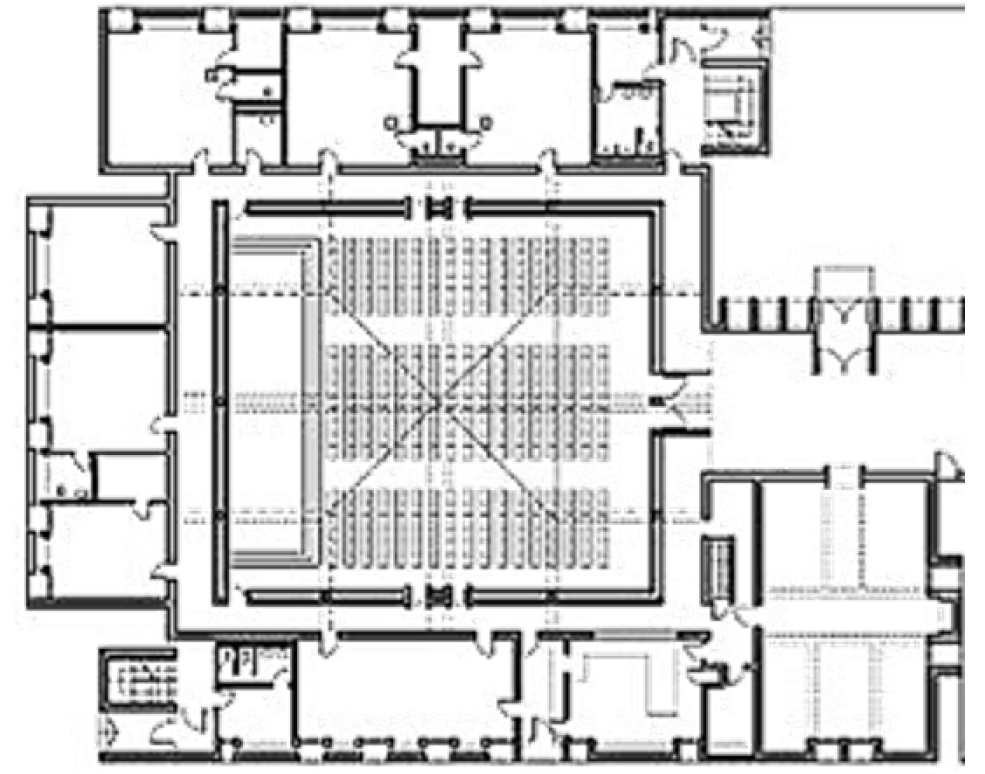

Por tal motivo, resulta adecuado mostrar el croquis conceptual elaborado por Kahn para la Primer Iglesia Unitaria de Rochester, el cual normalmente se oculta para mostrar la primera planta como si hubiera sido esa su primer idea. Si bien es cierto que algunos croquis que se muestran en revistas y de los que se encuentran con mayor facilidad tienen un aspecto muy cuidado e inclusive levantan sospechas de si fueron hechos luego de que la obra arquitectónica se terminó, es necesario darnos un derecho al asombro, al proceso que conlleva asombrarse y a no comprometer la obtención de resultados, por encima de la construcción de ideas.

¿Qué vamos a trazar si no hemos dedicado tiempo a pensar? Se asocia tan fuertemente a la Arquitectura con un "hacer" que no nos damos el espacio para la reflexión sobre lo que vamos a hacer, e inclusive olvidamos que hay un hacer en el pensar; el hacer interpretaciones, hacer asociaciones o crear ideas, son ejemplos de ello y son cuestiones primordiales para enfrentar el papel como una invitación y búsqueda más, dentro de nuestro proceso creador y no un momento frustrante y prematuramente obligado.

Nuestra capacidad de asombro debería de permitirnos notar cómo aquellos apuntes de primera mano y aquellos bocetos rápidos que hicimos para explicarnos algo a nosotros mismos o para dejar plasmada una idea son bases importantes de nuestro proceso y no hay nada de malo en que no tengan el aspecto de una obra maestra desde la primera vez. Son eslabones que se van transformando y traduciendo hasta que sea posible depositarlos fuera de nuestras mentes (en el papel, por ejemplo) para discutirlos con otras mentes.

Ahora bien, si se promueve que el estudiante deje de percibir el desarrollo de un proyecto como un encargo y se le permite explotar sus capacidades, sorprenderse y sorprender a los demás, es más probable que se sienta desobligado (en el buen sentido) e inspirado a buscar más experiencias. Guevara agrega:

(...) la sorpresa que interesa en este análisis, no es la del resultado, que es consecuencia; sino que el elemento a destacar es esa capacidad de sorprenderse con lo que dice la realidad, la admiración que despierta la realidad en el que crea.

El asombro ante la realidad es lo que sostiene el afán especulativo en el ser humano, la pregunta como evidencia del ser. Esa sorpresa es la cualidad que pertenece exclusivamente a la creatividad, significa esa relación especial del hombre con el mundo: buscar desinteresadamente, saber para poder ser. (Guevara, 2013, pp. 96-97) 


\section{El Experimento Progresista o Proceso Transformador: la reforma de la casualidad por la 'causalidad'}

En segunda instancia, nos referimos al experimento progresista, mas no por ello lo debemos comprender como un segundo paso para aprender sobre la Arquitectura. Se trata más bien de un complemento, en el que lo que nos asombra nos motiva a crear y experimentar, y viceversa.

No obstante, se debe considerar que la experiencia que surge de lo que se crea cobra sentido cuando da cuenta de que dejó una huella, fue aleccionadora o formó parte de una transformación. La experiencia por la experiencia en sí no es valiosa a menos que se le asigne una intención o que se le enrumbe hacia el progreso de una idea. El aprendizaje de la Arquitectura se orienta fuertemente a la puntualización de necesidades sociales que se pretenden resolver con la obra arquitectónica; pero si de aprender se trata, las vivencias en torno al proceso anterior a la resolución arquitectónica son las que, probablemente, transforman y validan el conocimiento.

Habría que recordar que el proyecto nunca tendrá una etapa más vivaz y llena de posibilidades que cuando está en el transcurso de su gestación, pues una vez terminado no es más que un resultado usualmente invariable.

Ante este planteamiento, el término proyecto no es más la obra terminada, sino todo un conjunto de hechos que se suceden hasta llegar a la obra terminada. Y lo mismo cuando se habla de diseño. El diseño no es la cosa en sí, son todas las etapas por las que se pasa para llegar a él. Es decir, que en la búsqueda del 'cognoscimiento' de la Arquitectura debería de pesar más el verbo que el sustantivo. No son 'el' proyecto, ni 'el' diseño, sino el 'yo-proyecto' y el 'yo-diseño' las herramientas esenciales. Es involucrar al estudiante en el proceso y en el entendimiento de que lo que hace no es un fin en sí mismo, sino una herramienta para vivenciar la Arquitectura y experimentar con ella.

Inclusive, a nivel lingüístico tiene un sentido de responsabilidad y apropiamiento: enfocarse en el hacer la obra, por encima de la obra hecha, pues desde el aprendizaje por resultados surgen los malentendidos de las obras que se describen como si se hubieran hecho solas, cuando en realidad hubo una mente creadora y pensante que trabajó, proyectó, diseñó, investigó y en general, fue el actor principal de un proceso.

Claro está que "(...) es más fácil dedicar tiempo y esfuerzo investigativo a las criaturas, en vez de a los creadores; a las obras creadas en lugar de a las operaciones de la mente creadora. Es más cómodo discutir sobre los resultados que reflexionar sobre las operaciones mentales que les dieron vida" (Guevara, 2013, p. 70).

Entonces, ¿qué se entiende por proceso? Un conjunto de actividades y eventos que se dan a lo largo del tiempo y pueden cambiar su orden, ser o no necesarios, o multiplicarse, dependiendo de la persona que los ejecute y la finalidad del proceso mismo. Cada actividad que se realiza durante el proceso deja huellas sobre lo que ha de ser, y son todas esas actividades las que se deben discutir en la academia, abiertamente, para el aprendizaje de todos.

En la mente del arquitecto está ya la idea de hablar del 'proceso de' diseño, 'proceso de' representación, 'proceso de' conceptualización, 'proceso de' pensamiento; pero todos ellos son algo demasiado específico como para ser el eje de aprendizaje en la academia. Por esto, es más beneficioso hablar de la 'dinámica del proceso', el procesoen-sí; no la creación, sino el acto de crear y todo lo que el estudiante experimenta mientras está creando.

Anhelar que un estudiante recolecte información y analice un sitio contemplando numerosas variables, más preocupado por el resultado que por las experiencias que pueda encontrar a lo largo del camino es como pedirle que aspire una gran bocanada de aire y la contenga hasta que sus energías y sus deseos de dar por terminada su represión lo lleven a exhalar el resultado. Sería más fructífero invitarlo a respirar y vivenciar cada etapa como un medio para potenciar las ideas.

Finalmente, todo lo que se investiga previamente antes de arrojar cualquier determinación no debería de ser visto como un momento anterior al comienzo del diseño arquitectónico, sino como una parte fundamental del proceso mismo. Guevara afirma que: "(...) la 
génesis y el desarrollo de un concepto tienen tanto valor proyectual, como el proceso de formalización y materialización que después le acontecerá" (Guevara, 2013, p. 47) y esta visión es un gran acercamiento a la importancia del proceso.

Sin embargo, cabe aclarar que dentro de la dinámica del proceso, se llega a soluciones nuevas y distintas, en la medida en que haya una provocación en la academia a experimentar y transformar lo existente, y si se utilizan discursos que entorpecen las experimentaciones de los estudiantes y que limitan su creatividad y su capacidad para defender su punto de vista, es muy probable llegar a resultados predecibles y con sabor a 'masdelomismo'. Con agudeza comenta el arquitecto y profesor Jorge Grané:

Estamos transitando momentos de desconcierto. Los estilos, los ismos y los paradigmas, que por tanto tiempo nos sirvieron de guía y respaldo, ya son parte de la historia que nos quisieron inculcar y son engavetados en cursos teóricos considerados, más que innovadores, restrictivos para la creatividad (Grané, 2013, p. 6).

Las ansias por llegar a respuestas concretas con rapidez han sido las fundadoras del divorcio mental entre el problema y la solución, que se visualizan como cuestiones independientes. Es común encontrar que, por ejemplo, a un proyecto académico de una casa unifamiliar, de un hospital o de cualquier tipo, se le llame problema de diseño como si el problema en sí, fuera diseñar el proyecto. Esto resulta en una reacción evasiva ante el proceso de diseño y una falsa percepción de que los problemas son algo que siempre está dado y ante lo que se plantea una determinada solución. Lo que un aprendizaje basado en la dinámica del proceso busca es comprender la solución dentro del mismo problema y no fuera de él: el resultado como parte de un proceso de problematización y experimentación.

De hecho, el error más común es suponer en vez de preguntar, pues significa que todo se asume sin cuestionamiento, sin intención indagadora, sin aprendizaje. Discutir, problematizar y cuestionar cargan con un valor muy negativo en la sociedad, como características de una actitud desafiante y prepotente, por lo que rápidamente el sistema educativo los oprime, cuando en verdad, son parte de la experimentación progresiva. No existe la 'serendipia', el accidente fortuito, o la coincidencia para quien sabe lo que anda buscando, pues mientras los estudiantes se esmeren en indagar respecto a sus intereses e intenciones, cada nuevo descubrimiento estará libre de casualidad y cada conocimiento, por simple que sea, podría ser revelador.

Paul Flory, quien recibió un premio Nobel de Química, en un discurso toma el fenómeno del descubrimiento fortuito y al respecto comenta:

(...) la casualidad normalmente juega una parte, eso es seguro, pero hay mucho más en la invención que la noción popular de venir caído del cielo. El conocimiento en profundidad y extensión son prerrequisitos indispensables. [Y además advierte:] A menos que la mente esté concienzudamente cargada de antemano, la proverbial chispa del genio, si se llegara a manifestar, probablemente no encontraría nada que prender. (Flory, citado en Romo, 1997, pp. 43-44)

Estas palabras atestiguan la necesidad de conocer los intereses con antelación, ahondar en ellos y, sobre todo, convertirlos en un continuo de procesos de asombro y experimentación que acabarán aumentando las posibilidades de hacer descubrimientos. El proceso es un relato de nuestra experimentación; relata los hallazgos, los retrocesos y cada una de nuestras ideas. Es una forma de atestiguar el progreso y de regresar en el tiempo para corregir o replantear ideas.

El escritor Matteo Corradini, en sus reflexiones sobre el desarrollo de la mente creativa, menciona: "Disfrutar el proceso es la primera sensación positiva del creador, que disfruta imaginando, en ese actuar movido por la fantasía, aún antes de verla realizada. El trayecto es importante porque es la sede de las acciones mentales creadoras" (Corradini, 2011, p. 31).

Es en la dinámica del proceso que adquirimos experiencias, lo que aumenta nuestros recursos para asimilar la realidad y poder transformarla. Si no se validan las experiencias como forma de aprendizaje, no habría ningún descubrimiento y seríamos incapaces de cuestionar y dirigir el destino de nuestros proyectos. Las experiencias contribuyen a agrandar nuestro repertorio, conocer otros puntos de vista y madurar nuestro semillero de ideas. Todo esto, finalmente, nos da la autoridad para reconocer nuestras 
intenciones durante la toma de decisiones y adueñarnos de la dinámica del proceso más plenamente.

\section{El Descubrimiento Compartido}

Parece ser una cuestión casi predecible que en un ambiente donde se busca aprender haya una gran accesibilidad a la información valiosa y se compartan los conocimientos; sin embargo, no lo es tanto. Históricamente, los conocimientos sobre Arquitectura eran algo que viajaba como en una especie de dinastía; de maestros a discípulos como una cuestión muy privilegiada y restringida. "La transmisión del saber se cuidaba y mantenía en secreto, lo cual con seguridad contribuía a que el proceso innovador fuera muy lento" (Guevara, 2013, p. 124). Luego, debido al desarrollo tecnológico y la masificación de la información, fue cada vez más difícil contener el conocimiento y los descubrimientos se hicieron mucho más accesibles.

En definitiva, cada proceso, por tratarse de una mutación iterativa, conduce a descubrimientos. Entonces, todas las experimentaciones cobran validez cuando a raíz de ellas se obtienen conclusiones y nuevos aprendizajes. Puede parecer a los ojos de otros que en los procesos creativos hay una repetición de las ideas, pero, en realidad, cada vez que se repite, si se cuenta con las bases del asombro y la experimentación progresista, las ideas van evolucionando y se van rectificando conceptos que, por lo común, solamente son observados por su creador.

En otros casos, gracias a la oportuna intervención de los profesores, habrá descubrimientos que surgirán sobre los procesos de revisión y diálogo estudianteprofesor. Pero también, casos en los que aprender y descubrir, dependerá en gran medida de las habilidades del estudiante para fijarse objetivos e investigar. El filósofo y pedagogo José Antonio Marina ha descrito estos procesos de exploración como algo muy paradójico, pero que se aproxima en mucho a lo que los estudiantes enfrentan, al decir:

Cada vez que un inventor, un científico o un artista se esfuerzan por realizar un proyecto, ha de comparar cada uno de sus pasos con el objetivo propuesto. Pero sucede que precisamente el objetivo es lo que se intenta encontrar, lo que se desconoce, con lo cual la búsqueda resulta dirigida por lo buscado, que al mismo tiempo es desconocido. (Marina, 1993, p. 164)

De esta manera, los estudiantes más perspicaces se dan cuenta de que no encontrarán en la academia las respuestas a todas sus preguntas de manera inmediata y que en ocasiones les hará falta más información sobre ciertos temas, pues no todo está dado. Es común que se soliciten ciertas habilidades sin que se explique con claridad cómo se obtienen, o que se asuma el conocimiento de ciertas teorías sin comprobar si de hecho el estudiante las conoce. Aún sin que la academia se percate demasiado de ello, entre los estudiantes de Arquitectura se promueve fuertemente desde los primeros años un compromiso de búsqueda y una gran capacidad autodidacta que les permite llevar los conocimientos de los talleres y las aulas varios pasos más lejos que si se conformaran con las pistas informativas que reciben en cada curso.

En el aprendizaje de la Arquitectura, son los pequeños (o grandes) descubrimientos que el estudiante hace, tal vez influenciado por algún curso, pero finalmente por su cuenta; los que lo llevan a elevar su compromiso hacia la profesión y valorar el camino recorrido. Por lo que:

(...) dotar a los alumnos -futuros arquitectos- de la necesidad de esta investigación hasta el infinito, es un objetivo claro de mejora social, no sólo por el producto de su trabajo como arquitectos sino además, y sobre todo, por la repercusión en la formación de su autonomía personal. (Álvarez \& Gómez, 2009, p.3)

Sin embargo, la investigación es algo que ocurre, en la mayoría de los casos, como una situación al margen del desenvolvimiento de los alumnos; cada persona desarrolla ideas como mejor le parece, pero aunque todos formen un único grupo de interés, que es la Arquitectura, pocas veces se comparten consejos o puntos de vista. Por esto, es también necesario dejar atrás el convencionalismo del profesor que revisa lo que sus alumnos hacen, y refrescar el conocimiento permitiendo que los estudiantes compartan 
Figura 4. Diagrama El Proceso de Conversación. Elaboración propia, basado en Wagensberg (2000). sus hallazgos, establezcan fortalezas y deficiencias en común y debatan entre ellos (en conjunto con los profesores) sobre sus quehaceres. Pues, tal y como lo señala la arquitecta, profesora e investigadora Cristina Dreifuss:

El no establecer un lenguaje común y un conjunto de códigos y criterios manejados, tanto por alumnos como por los profesores al interior del taller, plantea una brecha que deviene en producciones hechas 'a gusto del profesor', que se alejan de un espíritu de búsqueda creativa por parte del alumno. (Dreifuss, 2008, p. 27)

Además, "la calidad de un obra depende de la calidad del criterio, pero ¿quién nos advierte sobre la calidad del propio criterio?" (Marina, 1993, p. 204). En respuesta a esta interrogante, debemos comprender que el criterio en sí mismo se construye en un proceso individual y colectivo. Así, los descubrimientos que se comparten intensifican las experiencias en torno al 'cognoscimiento' de la Arquitectura porque admiten el encuentro de criterios. De manera que, entre mayor sea el grado de exposición de los descubrimientos, se induce al estudiante a confrontar una mayor cantidad de criterios, de los cuales pueden surgir nuevas ideas o grupos de interés que mejoren y continúen desarrollando lo expuesto.

Entonces, el aprendizaje de la Arquitectura se registra como un conjunto de experiencias, habilidades y procesos creativos que se desarrollan a lo largo del tiempo. Sin embargo, la intención principal no debe ser el proyecto por el proyecto, la tarea por la tarea, y, mucho menos, la evaluación por la evaluación. Un proyecto de investigación, una Escuela de Arquitectura, una exposición, una conferencia, un texto, sólo son algo si estimulan el diálogo. De esta manera, compartir los descubrimientos es algo vital para enriquecer y multiplicar el aprendizaje que surge a raíz de 'cognoscer' la Arquitectura.

\section{La conversación: un medio para activar las bases del aprendizaje}

Las tres bases del aprendizaje, sobre las que hemos hablado; están íntimamente relacionadas con los procesos de conversación; y fundamentalmente si somos conversadores virtuosos (ver figura 4) se nos abrirán las puertas de un amplio universo de argumentaciones, discusiones e información que enriquecerá nuestro manejo de las ideas. A través de este diagrama se concibe un diálogo con nosotros mismos, con la realidad y con el otro, como un modo de comprender que asombrarse, experimentar y compartir descubrimientos son actividades complementarias entre sí, que regeneran los significados de la Arquitectura.

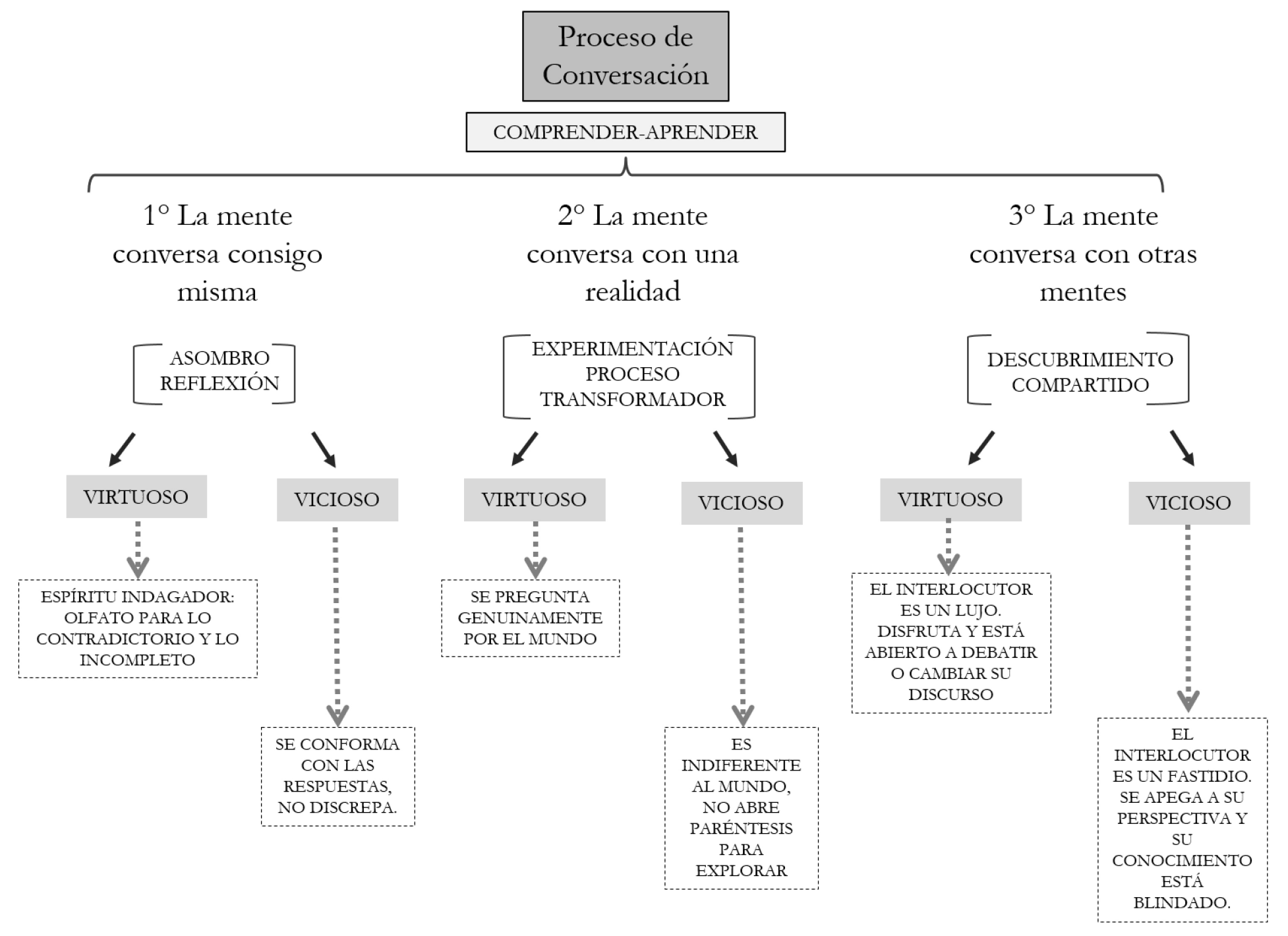


Figura 5. Diagrama Yo-Proyecto (enfocado en la idea del Yo). Elaboración propia.
En definitiva, nuestras conversaciones son gestoras de nuevos conocimientos, pues cuando leemos un libro, escribimos un ensayo, hacemos una exposición o creamos un croquis bajo la intención de aprovechar todas las virtudes de esa conversación uni- o pluri-dimensional, obtenemos un resultado aún más gratificante y con mejores posibilidades de desarrollo que cuando dejamos estas oportunidades pasar, sin caer en cuenta de que son herramientas de aprendizaje.

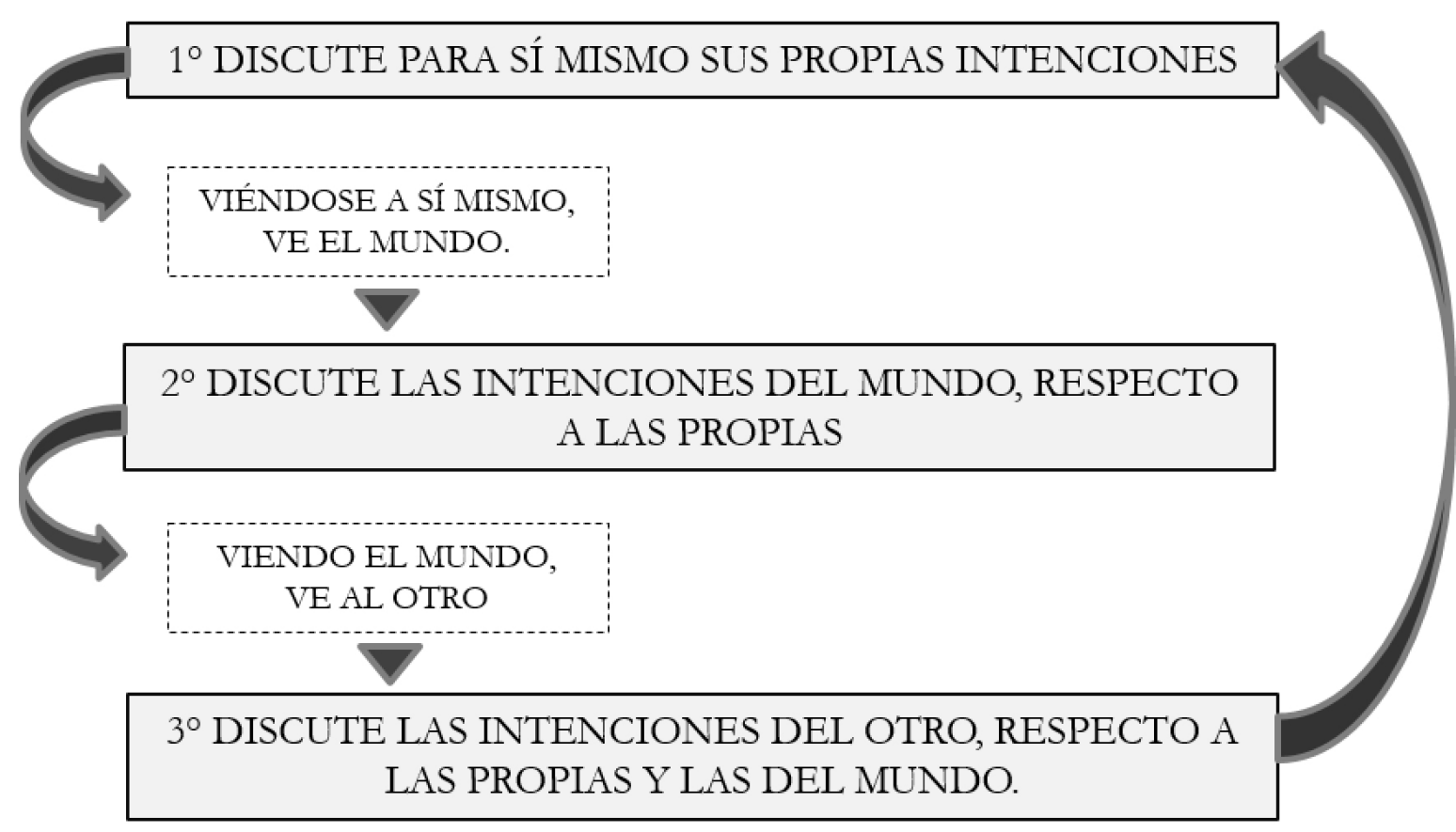

Así, según la figura 5, se podría concebir el 'cognoscimiento' de la Arquitectura como una propuesta para la formación de personas/profesionales que aprenden y desarrollan ideas arquitectónicas con la motivación de reformar teorías y prácticas, o aportar nuevos descubrimientos; que se interesan más por las preguntas y las negociaciones que por las respuestas y las afirmaciones; y cuya intención no se reduce a aprender únicamente desde la percepción del yo, sino expandiendo sus experiencias a través de la afirmación del mundo y los otros como potenciales interlocutores que reaniman la intención primera.

Entonces, se podría decir que si anteriormente hablamos del Yo-Proyecto desde una perspectiva más general y enfocada en la idea de Proyecto (ver figura 2), esta figura (5) vendría a ser una visión del Yo, más detallada e introspectiva que explica el sentido de aprender desde las bases del 'cognoscimiento' como modos de actuar elementales para crear nuevas ideas e instar a los estudiantes a desarrollar con emprendedurismo sus intenciones como personas/profesionales.

En general, el aprendizaje de la Arquitectura parece ir encontrando poco a poco una armonía cada vez mayor con las necesidades reales de la sociedad y con las expectativas en cuanto a conocimientos técnicos. Pero se debe recordar que si bien es cierto que los asuntos informáticos, constructivos, administrativos y demás complementos técnicos son parte de la formación de los arquitectos, no son necesariamente estas las cuestiones más enriquecedoras a nivel de aprendizaje. Steven Holl en una entrevista realizada por Moya-Angeler afirma que:

(...) las preguntas filosóficas son también urgentes. Necesitamos poesía tanto como necesitamos pan. [Y agrega] Deseo trabajar en las cuestiones filosóficas y elevarlas al nivel de la necesidad. El construir una analogía, elaborar un concepto o una idea poética sobre él, provoca la integridad del proceso. Construir un edificio es una batalla; todas las personas involucradas están constantemente intentando tirar hacia su lado. Uno necesita la fuerza de un concepto poético. Y eso es lo que intento transmitir a mis alumnos.

Recuerdo muchos talleres de proyectos que se basan en enseñar una determinada técnica. Esto es lo que falla en la Arquitectura actual: esa euforia desmedida de la técnica en vez del pensamiento. Necesitamos elevar la disciplina de la Arquitectura al nivel del pensamiento filosófico. (Moya-Angeler, 2007, pp. 85-86)

Claramente, Holl invita a crear un equilibrio entre el aprendizaje de la técnica y la reflexión profunda; pero sí hace hincapié sobre el hecho de que, en la actualidad, se tiende a enseñar técnica por encima de pensamiento, y que eso afecta inmensamente la posibilidad de construir nuevas teorías y resoluciones prácticas a las problemáticas que atañen al quehacer arquitectónico. 
Dichosamente, aunque en ocasiones se olvidan o desestiman los valores cognitivos y vivenciales como modos de aprender sobre la Arquitectura, es reconocible la existencia de un espíritu sensible hacia todas ellas, y en especial, hacia el asombro, ya que es gracias a esta tendencia a cuestionar y problematizar sobre los hechos cotidianos que los arquitectos se adentran en una dimensión alterna de la realidad, en la cual encuentran cabida la creatividad y la innovación como modos de exacerbar los significados de las cosas en las situaciones más rutinarias.

Por lo tanto, si continuamos desarrollando el asombro como motor del conocimiento, seremos capaces de encontrar y proponer nuevas problemáticas; si encontramos nuevas problemáticas y las insertamos en experimentos creativos que nos permitan hacer descubrimientos, tendremos propuestas innovadoras; y si todo ese conocimiento se hace colectivo y entra en diálogo con otras propuestas, tendremos ideas emergentes que motivarían a que el tránsito por la Escuela de Arquitectura, más que una serie de conocimientos adquiridos tras el cumplimiento de la malla curricular, sean una suma de experiencias significativas que garantizan que siempre habrá 'un más allá' para la Arquitectura.

\section{Referencias Bibliográficas}

Álvarez, E \& Gómez, C. (2009). Crítica Radical a la Enseñanza de la Arquitectura: Algunas Precisiones sobre los Términos "Investigación" y "Proyecto". São Paulo: IV Projetar.

Aristóteles. (1994). Metafísica. Madrid: Editorial Gredos.

Castaño, J., Bernal, M., Cardona, D \& Ramírez, I. (2005). La Enseñanza de laArquitectura: Una Mirada Crítica. Revista Latinoamericana de Estudios Educativos. 1, 125-147.

Conte-pomi, G. (2009). El País Fértil. Notas para una Pedagogía del Proyecto. Tesis Doctoral. Universidad Politécnica de Cataluña.

Corradini, M. (2011). Crear Como se Desarrolla una Mente Creativa. Madrid: Narcea.

Dreifuss, C. (2008). La Arquitectura en su Enseñanza/Aprendizaje en el Primer Taller de Diseño. Universidad Nacional de Ingeniería, Facultad de Arquitectura, Urbanismo y Arte, Lima, Perú. Recuperado de: http://www.bibliotecacentral.uni.edu.pe/pdfs/ CIUDADYARQUITECTURA/1,2008/art_0002.pdf

Grané, J. (2013). Las Ciencias Cognoscitivas Aplicadas a la Enseñanza de la Arquitectura: Hacia una Definición de la Arquitectura. RevistArquis, 2(1), 1-14.

Guevara, O. (2013). Análisis del Proceso de Enseñanza Aprendizaje de la Disciplina Proyecto Arquitectónico, en la Carrera de Arquitectura, en el Contexto del Aula. Universidad Autónoma de Barcelona, Tesis del Programa de Doctorado: Calidad y Procesos de Innovación Educativa, Departamento de Pedagogía Aplicada.

Heidegger, M. (2001). Introducción a la Metafísica. Barcelona: Editorial Gedisa.

Linares, A. (2006). La Enseñanza de la Arquitectura como Poética. Barcelona: Ed. UPC (Universidad Politécnica de Cataluña)

Marina, J. (1993). Teoría de la Inteligencia Creadora. Barcelona: Editorial Anagrama.

Moya-Angeler, E. (2007) Una Conversación Académica con Steven Holl. Estrategias de Formación. Revista Arquitectos, 180(1), 84-86

Piñón, H. (8 de octubre, 2007). Reflexión sobre la Docencia de la Arquitectura. Revista Vitruvius, (89). Recuperado de: http://www.vitruvius.com.br/revistas/read/ arquitextos/08.089/195

Platón. (1871). Obras Completas: Teeteto (Tomo 3) Madrid: Ed. de Patricio de Azcárate

Poe, E. (1846). The Philosophy of Composition. (Masís, A. trad.). Graham's Magazine, 18. Versión en línea. Recuperado de: http://www.catedras.fsoc.uba.ar/reale/ filosofia-de-la-composicion-poe.pdf

Real Academia Española. (2014). Diccionario de la Lengua Española (23.a ed.). Recuperado de: http://dle.rae.es/?w=diccionario 
Romo, M. (1997). Psicología de la Creatividad. Barcelona: Paidós.

Szymborska, W. (2009). Número Equivocado y Otros Poemas. República Dominicana: Muestrario de Poesía.

Wagensberg, J. (4 de octubre de 2000). Tribuna: Conversar, Conversar. Periódico El País, Edición América. Recuperado de: http://elpais.com/diario/2000/10/04/ futuro/970610410_850215.html 